\title{
Aprendiendo a investigar: Analizando la problemática en torno al trabajo final de la Licenciatura en Ciencia y Tecnología de Alimentos
}

\author{
Learning to investigate: An approach to the problem in the final work \\ of the Bachelor in Food Science and Technology
}

\author{
Mariela Patrignani \\ https://orcid.org/0000-0001-5280-7779 \\ mariela_patrignani@hotmail.com \\ Universidad Nacional de La Plata
}

\section{RESUMEN}

La mayor parte de los programas institucionales de apoyo y contención estudiantil se centralizan en los primeros años de las carreras universitarias, y buscan minimizar la deserción en los trayectos iniciales de la formación. Sin embargo, la cantidad de estudiantes que abandonan los estudios en los últimos años no es menos importante y entre los motivos de esta tendencia se destaca las demoras en la producción del trabajo final.

Si bien existen pocos trabajos enfocados en el trabajo final de carrera, el interés en este tema de investigación aumenta cuando reconoce que el mismo genera un alto grado de estrés no solo para los estudiantes sino también para docentes. Si a este factor se le suma la falta de valoración de los motivos por los cuales se debe hacer una Tesis o Tesina, el trabajo final de carrera puede representar en muchos casos un verdadero obstáculo limitando así los tiempos de egreso. El presente trabajo busca contribuir al análisis de trabajos finales de licenciatura y aportar, no solo a una sistematización de autores referentes en el tema, sino también a una propuesta concreta de revisión curricular de la Licenciatura en Ciencia y Tecnología de los Alimentos de la UNLP, carrera utilizada como ejemplo de análisis.

\section{ABSTRACT}

Most of the programs for student retention are centralized in the early years of the career, and seek to minimize dropouts in the initial training paths. However, the number of students who desert in the last years of their career is also important. Among the reasons for this trend, the delay in the production of the final work can be highlighted.

Although there are few researches focused on the final work of the career, the interest in this topic increases when it is recognized that it generates a high degree of stress not only for students but also for teachers. Besides, if we add the lack of assessment for which this work must be done, the final career work can represent a real obstacle. The aim of the present work is to contribute to the analysis of final work of the career and contribute not only to a systematization of authors, but also to a specific proposal for a curricular review of the Bachelor in Food Science and Technology (career used as an example of analysis).

\section{PALABRAS CLAVE}

Trabajo final de carrera, trayectorias, alfabetización académica, egreso universitario

\section{KEY WORDS}

educational work, university, curricula teaching, tutorial classes. 


\section{PRESENTACIÓN}

En los últimos años el paso de la universidad de élite a la universidad de masas sumado a las vicisitudes del mercado laboral, generaron notables transformaciones en la vida de los estudiantes ${ }^{1}$. La figura de un estudiante de tiempo completo fue paulatinamente reemplazada por la del estudiante dividido entre la institución universitaria y el mundo del trabajo. A partir del siglo XXI también deben incluirse la aparición de las herramientas del universo de las TICs (tecnologías de información y comunicación). El impacto de estas herramientas se manifiesta en las producciones y en las prácticas comunicativas de los jóvenes quienes son poseedores de nuevos saberes no reconocidos por la institución universitaria (Casco, 2007).

El ingreso a la universidad implica que el recién llegado aprenda su oficio de estudiante. Este tránsito significa la ruptura con su pasado inmediato y el enfrentamiento con un futuro todavía opaco. El proceso implica tres etapas: la alienación (entrada a un universo desconocido que rompe con el mundo anterior); el tiempo del aprendizaje (definición de estrategias y adaptación); y el tiempo de la afiliación (adquisición de un relativo dominio de las reglas institucionales) (Casco, 2007). Si este pasaje es exitoso, el individuo avanza desde su condición de novato a la condición de aprendiz, y de ella a la de miembro afiliado. La metáfora "oficio de estudiante" resalta el carácter no natural ni espontáneo del nuevo estatus que deberá alcanzar el ingresante. En su trayectoria, el estudiante articula una manera de ser joven y una relación con los estudios. Esto, sumado al proceso de ampliación de la matrícula universitaria de los últimos años, dan cuenta de la heterogeneidad de sujetos ingresantes (Ros, Benito, Germain, Justianovich, 2017). Las trayectorias ya no pueden pensarse desde la homogeneidad de los sujetos universitarios, sino como serie de las posiciones su-

En el presente trabajo se utilizan términos masculinos (los estudiantes, los tesistas, los directores, los talleristas, etc.) como una forma nombrar a los sujetos con el único objetivo de facilitar la lectura. Sostenemos, sin embargo, la necesidad de revisar y ajustar el lenguaje para encontrar métodos satisfactorios de visibilizar tanto a las mujeres como a otros géneros no binarios. 
cesivamente ocupadas por un mismo grupo en un espacio sometido a incesantes transformaciones (Pierella, 2014). Pensar las trayectorias desde una visión integral posibilita el diseño de estrategias de acompañamiento que fomenten la permanencia, el egreso, y disminuyan el abandono universitario (Ros et al., 2017).

\section{La metáfora "oficio de estudiante" resalta el carácter no na- tural ni espontáneo del nuevo estatus que deberá alcanzar el ingresante. En su trayectoria, el estudiante articula una manera de ser joven y una relación con los estudios.}

Las estrategias para abordar la problemática de la deserción deben entender las trayectorias estudiantiles y su contexto socio-histórico, cultural e institucional. No tendrán los mismos recorridos académicos estudiantes que han tenido acceso diferencial a determinados bienes materiales y simbólicos que aquellos que han tenido que sortear más de un obstáculo para continuar su proceso de formación. Es por esto que para asegurar el acceso democrático se debe ofrecer a los estudiantes las mejores herramientas y mecanismos para la apropiación del saber desde el comienzo hasta el final de su vida académica.

Considerando el modelo de integración del estudiante, cuanto mayor sea el vínculo del ingresante con el ambiente académico, mayor será su compromiso con la institución y menor su probabilidad de descontinuar su vida académica. Es por esto que la mayor parte de los programas de apoyo y contención estudiantil se centralizan en los primeros años de la carrera, y buscan minimizar la deserción en los trayectos iniciales de la formación. Sin embargo, la cantidad de estudiantes que abandonan los estudios en los últimos años no es menos importante y entre los motivos de esta tendencia se destaca las demoras en la producción del trabajo final (requisito final para graduarse de casi la mitad de las carreras ofrecidas por la UNLP).

\section{Considerando el modelo de integración del estudiante, cuanto mayor sea el vínculo del ingresante con el ambiente académico, mayor será su compromiso con la institución y menor su probabilidad de descontinuar su vida académica.}

Si bien existen pocos trabajos enfocados en el trabajo final de carrera, el interés en este tema de investigación aumenta cuando reconoce que el mismo genera un alto grado de estrés no solo para los estudiantes sino también para docentes (Rotstein, De Gueller y Soláns, 
2014). Si a este factor se le suma la falta de valoración de los motivos por los cuales se debe hacer una Tesis o Tesina, el trabajo final de carrera puede representar en muchos casos un verdadero obstáculo limitando así los tiempos de egreso (Gascón, 2008). La posibilidad para que esta situación se revierta se basa en el compromiso de toda la comunidad universitaria.

El presente trabajo forma parte de mi trabajo final de Especialización en Docencia Universitaria, que tuvo como objetivo central fomentar el egreso de los estudiantes en las últimas etapas de su formación. Esta primera sección busca contribuir al análisis de trabajos finales de licenciatura y aportar no solo a una sistematización de autores referentes en el tema, sino también a una propuesta concreta de revisión curricular de la Licenciatura en Ciencia y Tecnología de los Alimentos de la UNLP, carrera utilizada como ejemplo de análisis. De este modo, en una segunda parte, se propondrá una alternativa que posibilite el acompañamiento de los estudiantes en los últimos años de la carrera.

\section{TRAYECTORIAS ESTUDIANTILES EN LA LIC. EN CIENCIA Y TECNOLOGÍA DE ALIMENTOS}

Las trayectorias estudiantiles y sus problemáticas sólo pueden entenderse desde el contexto en donde ocurren. La Lic. en Ciencia y Tecnología de Alimentos comenzó a dictarse en el 2002 en la Universidad Nacional de La Plata. Esta carrera cuenta con no más de 50 ingresantes por año, esto asegura grupos reducidos en los últimos años y un trato cercano con los docentes.

De acuerdo al plan de estudios, la Lic. en Alimentos cuenta con 35 materias y un trabajo final que debe ser realizado en el plazo de un semestre académico en paralelo con otras 3 materias. Sin embargo, rara vez se cumplen estos plazos. En el año 2017 la duración promedio de la Lic. en Alimentos fue de más de 8 años, superando incluso los tiempos de egreso de la carrera de bioquímica (carrera de 6 años según su plan de estudios).

En el marco del Programa de Egreso de la facultad de Ciencias Exactas, en el año 2016 se buscó identificar las principales dificultades de los estudiantes de la Lic. en Ciencia y Tecnología de los Alimentos. De acuerdo a los datos recopilados ${ }^{2}$, se reafirmó la percepción general de que la duración de los trabajos finales de grado de la Lic. en Ciencia y Tecnología de Alimentos exceden los tiempos establecidos en los planes de estudio. Se pudo concluir que en las condiciones actuales a los estudiantes de la Lic. en Ciencia y Tecnología de Alimentos les es prácticamente imposible concretar el trabajo final en los plazos establecidos. Esto, por supuesto, trae aparejado un importante peso psicológico.

Presentados en la Revista Anual de la Facultad de Cs. Exactas (2017) y en informes confeccionados por el Programa de Egreso. 
En el marco del Programa de Egreso de la facultad de Ciencias Exactas, en el año 2016 se buscó identificar las principales dificultades de los estudiantes de la Lic. en Ciencia y Tecnología de los Alimentos. De acuerdo a los datos recopilados, se reafirmó la percepción general de que la duración de los trabajos finales de grado de la Lic. en Ciencia y Tecnología de Alimentos exceden los tiempos establecidos en los planes de estudio.

La Lic. M. Florencia Lopez es tutora de trabajos finales y desde agosto del 2018 trabaja en conjunto con el programa de egreso. Ella ha podido detectar que una de las principales problemáticas es el tiempo que tardan los estudiantes en finalizar el trabajo final (el tiempo real se calcula en 1 año y medio, muy superior a los 6 meses estipulados por los planes de estudio). Por otra parte, parece claro que los problemas y tensiones más manifiestos se presentan en la etapa de escritura. Esto estaría relacionado a la escasa alfabetización académica con la que cuentan los estudiantes. A pesar de que los tesinistas están acostumbrados a presentar informes cortos, desconocen el formato real y el lenguaje utilizado en la redacción de un texto largo. Por otra parte, no es habitual para ellos recibir una corrección formal del trabajo realizado. Esto enlentece el proceso de producción del trabajo final y puede incluso llevar a conflictos con los directores o tutores (Patrignani, 2019).

\section{LA ENSEÑANZA DEL PENSAMIENTO CIENTÍFICO EN EL MARCO DE LAS CIENCIAS EXACTAS}

A partir de un análisis centrado en las prácticas pedagógicas, advertimos que si bien los estudiantes en la Facultad de Ciencias Exactas cuentan con actividades prácticas durante las cursadas regulares, el objetivo de las mismas es corroborar hipótesis o buscar la aplicación/demostración de aquello que se dio en la teoría, no la generación de un proceso autónomo de análisis. Asimismo, si bien en algunas materias se exige la presentación de un informe de laboratorio, este texto es del tipo descriptivo y carece de las estructuras argumentativas típicas de los documentos científicos.

En este sentido, la enseñanza de las ciencias desde su propia metodología ha quedado reducida a versiones muy simplistas (Gil Pérez, 1983). Desde una mirada crítica, principalmente en los primeros años de la carrera, no se plantean actividades de investigación experimental acompañados de actividades de lectura y escritura. Parecería que la práctica investigativa, ha quedado restringida únicamente al desarrollo del trabajo final de carrera. Esto probablemente 
responde a que, debido a la masividad del estudiantado, son pocos los casos en los que para acreditar la promoción se incluya la realización de ensayos o monografías individuales en donde el estudiante deba redactar un texto riguroso, preciso estructurado y con coherencia valiéndose de distintas herramientas de indagación y análisis. De aquí se despliega que, si bien la educación universitaria busca asegurar la formación científica, el saber crítico no es algo que se ejercite activamente durante el paso de los estudiantes por las casas de altos estudios. El paradigma de la enseñanza de las ciencias acorde al proceso de producción de conocimientos, exige investigaciones concretas y la familiarización de los estudiantes con el método científico (Gil Pérez, 1983).

Esta situación obliga a repensar el papel de las instituciones en conjunto con un análisis de las situaciones a las que son sujetos habitualmente los estudiantes universitarios.

\section{EL CURRICULUM OCULTO DETRÁS DEL TRABAJO FINAL DE LA LIC. EN ALIMENTOS}

De acuerdo con el Reglamento de trabajos finales de la Licenciatura en Ciencia y Tecnología de los Alimentos el trabajo final deberá:

Constituir una instancia formativa que abarque uno o más aspectos relacionados con la carrera y las proyecciones en la vida académica y/o profesional del futuro egresado. Teniendo en cuenta que el motivo fundamental de la inclusión de un trabajo de estas características en el plan de estudios es la formación del estudiante, se requerirá que las tareas a desarrollar permitan realizar un análisis de situación/estado del arte sobre el sistema/problema de estudio, analizar críticamente los resultados obtenidos y extraer conclusiones sobre el trabajo realizado bajo la guía de su(s) director(es)" (reglamento del trabajo final de la Licenciatura en Ciencia y Tecnología de los Alimentos, 2018, p. 1)

Parece claro que el trabajo final en la carrera de grado, definido de esta forma, tiene como meta fomentar el desarrollo del pensamiento y de las habilidades del estudiante para que el mismo tenga un manejo crítico y reflexivo de la información utilizando como propuesta metodológica el método inductivo de investigación didáctica. El aprendizaje adquirido en este modelo se supone capaz de desarrollar modos de pensar y de manejo de información de forma sistemática que permitan aplicar los conocimientos ya adquiridos de manera eficaz (Davini, 2008).

Sin embargo, las actividades que fomentan este pensamiento crí- 
tico no se desarrollan dentro de la estructura universitaria, sino que quedan relegados a espacios subalternos de formación por fuera de la estructura curricular e implican el compromiso de un director que no necesariamente es un docente (Arroupe, 2015). Estos directores deben contar con el espacio y los recursos económicos para que los estudiantes desarrollen las labores experimentales, lo que muchas veces enlentece el proceso de egreso. Además, en el esquema actual, la tarea de aportar las herramientas para la redacción del trabajo final recae sobre el director, a causa de la escasa alfabetización académica con la que cuentan los estudiantes al finalizar las cursadas regulares. Esto dificulta aún más su labor y puede llevar a tensiones con los mismos estudiantes ya que debe cumplir funciones que deberían ser resueltas dentro de la misma institución universitaria.

Si pensamos al trabajo final de carrera como una materia más de un plan de estudios con una estructura ya pactada, sería de esperar que el mismo contara con un currículo, un plan de estudio y un programa. Sin embargo, estos documentos curriculares que contienen los elementos conformantes de la propuesta de una materia, parecen estar ausentes. No obstante, no parece haber dudas que el producto final de esta materia sí cumple con ciertas perspectivas implícitas.

El trabajo final, a diferencia del resto de las materias de grado, parece operar enteramente a partir del currículum oculto ${ }^{3}$ que implícitamente establece los modos de hacer las cosas y los requisitos a cumplir (Barco, 2016). Este currículum, tal como describió Barco (2016) se desarrolla en forma tácita entre el docente que dirige y el estudiante (o en este caso tesinista) (Arroupe, 2015). Ejemplo de ello es el tipo de escritura que se debe desarrollar, en donde, hay exigencias del modo en que se debe hacer, pero"la escritura" como contenido de enseñanza no siempre se explicita; se evalúa (en tanto exigencia del código académico) pero no siempre se enseña.

Para poner de manifiesto la estructura del trabajo final se seleccionaron 10 trabajos presentados entre el año 2013 y el año 2018. Estos trabajos finales comparten un lenguaje específico, formal e impersonal. Los términos utilizados son precisos y se evitan palabras ambiguas. Expresiones coloquiales como "en mi opinión" o "podría ser..." son descartadas y reemplazadas por otras como "se podría concluir..." o "se puede deducir"; además los conectores utilizados e incluso los tiempos verbales (la introducción en presente mientras que el resto del manuscrito se escribe en pasado) responden a este género aca-

3 El curriculum oculto hace referencia a los aprendizajes dentro de la esfera de lo actitudinal y valoral. Este tipo de curriculum es una forma de socialización y adaptación y nos habla de las conductas que se desarrollan como parte del "aprendizaje colateral" dentro del curriculum explicito (Santomé, 1991; Díaz Barriga, 2006). 
démico particular para una audiencia especializada. En entrevistas realizadas por la Lic. Arroupe a estudiantes tesinistas se deja entrever el proceso de adaptación por el que transitan los estudiantes al sistematizar su pensamiento y el "aprendizaje de esas reglas y normas que parecen restringir el pensamiento" (Arroupe, 2015, p. 16). De acuerdo a Hernández Zamora (2009), esta postura cerrada impuesta en la forma de escritura y ajena al contexto sociocultural del estudiante tiende a excluir a los miembros de sectores históricamente marginados.

\section{EL ACOMPAÑAMIENTO PEDAGÓGICO DURANTE EL DESARROLLO DEL TRABAJO FINAL}

Actualmente existe una escasa relación entre la modalidad de inserción de las tesinas y las materias teórico-metodológicas de los planes de estudios (Arroupe, 2015). Sin el acompañamiento adecuado, la escritura, desanima y conduce a la postergación o incluso hasta la autoexclusión de algunos estudiantes quienes no se sienten preparados para la tarea (Pereira y Di Stefano, 2007; Hernández Zamora, 2009; Arroupe, 2015).

Por otra parte, el uso de instructivos referidos a cómo escribir una tesis no conducen a que los estudiantes se interroguen sobre cuestiones más allá de la distribución de la misma o la presentación de la información. No hay un espacio ni medios para reflexionar sobre la propia producción a partir de un trabajo interactivo entre los participantes. Esto va en contra a lo que la psicología del desarrollo considera como aprendizaje efectivo "a través de un proceso colaborativo, de interacción entre docentes y aprendices que van internalizando paulatinamente los conocimientos y habilidades que son objeto de enseñanza hasta alcanzar la autonomía" (Pereira y Di Stefano, 2007, p. 211).

De acuerdo a Gil Pérez (1983) la enseñanza de la ciencia tiene un carácter social y no debe quedar reducida a la asimilación del discurso profesional ni a la interacción estudiante-profesor, requiere de la actividad colectiva y del trabajo en equipo.

\section{CONSIDERACIONES FINALES}

Las problemáticas en torno al trabajo final de la Lic. en Ciencia y Tecnología de Alimentos son variadas y complejas. Por un lado, durante el transcurso de la vida académica los estudiantes cuentan con limitados espacios en donde se promueva y ejercite el pensamiento crítico, quedando éste postergado al último momento antes de la graduación. Durante esta última etapa el estudiante también debe adquirir las herramientas discursivas para ingresar a la comunidad académica y ser reconocido como un par. Estas capacidades, si bien son evaluadas, no son enseñadas durante la carrera de grado y resulta en importantes 
dificultades durante la redacción del trabajo final. Por otra parte, aunque el trabajo final esté incluido como una materia de grado, el mismo opera enteramente bajo un curriculum oculto cuyas normas parecen ser sólo conocidas por la esfera académica, quienes luego avalarán o no la pertenencia del aprendiz a su comunidad. Finalmente se destaca también la escasez de espacios en donde el quehacer científico se vea como una actividad social durante la carrera de grado.

Como docentes universitarios quizá deberíamos preguntarnos acerca de las oportunidades que les damos a los estudiantes para la apropiación de los contenidos (más allá de la mera repetición de los mismos); qué tiempo real se le da a la escritura y a la enseñanza de la misma durante las cursadas regulares; qué intervenciones realizamos para generar mejores condiciones de aprendizaje para los estudiantes; qué alternativas buscamos para resolver los problemas de redacción con los que se encuentran los estudiantes al ingresar a la Universidad; cuáles serían los posibles canales de comunicación entre las materias para que las mismas dejen de funcionar como compartimientos estancos; si la alfabetización es un proceso (no un estado) qué herramientas les estamos aportando los futuros profesionales para poder leer y escribir en su disciplina particular. Los docentes tenemos una gran responsabilidad no solo académica sino social sobre la formación de los profesionales.

Considerando todo esto, en una segunda parte de este trabajo ${ }^{4}$ se describirá una propuesta de innovación pedagógica diseñada para que el trabajo final recorra de forma transversal los últimos años de la carrera de grado, favoreciendo el acercamiento del estudiante a la alfabetización académica como tesista y como futuro profesional.

qué tiempo real se le da a la escritura y a la enseñanza de la misma durante las cursadas regulares; qué intervenciones realizamos para generar mejores condiciones de aprendizaje para los estudiantes; qué alternativas buscamos para resolver los problemas de redacción con los que se encuentran los estudiantes al ingresar a la Universidad; cuáles serían los posibles canales de comunicación entre las materias para que las mismas dejen de funcionar como compartimientos estancos; si la alfabetización es un proceso (no un estado) qué herramientas les estamos aportando los futuros profesionales para poder leer y escribir en su disciplina particular.

\footnotetext{
$4 \quad$ Agradecimientos: a la Universidad Nacional de La Plata y a todo el equipo de la Especialización en Docencia Universitaria por darme la oportunidad de perfeccionarme como docente, dando un lugar a la reflexión de las propias prácticas. Además, quisiera agradecer muy especialmente al director de mi TFI de la Especialización Santiago Zemaitis por su apoyo en este proyecto y por su entusiasmo desde un primer momento.
} 


\section{BIBLIOGRAFÍA}

Arroupe, N. (2015). "El desarrollo de trabajos finales: escenarios y relación con el saber en las trayectorias académicas de los estudiantes de grado". Síntesis, (4), 1-22.

Barco, S. (2016). "Glosario del seminario de Desarrollo e innovación curricular". Manuscrito no publicado, Universidad Nacional de La Plata, Argentina.

Casco, M. (2007). "Prácticas comunicativas del ingresante y afiliación intelectual". En V Encuentro Nacional y II Latinoamericano "La universidad como objeto de investigación". Facultad de Ciencias Sociales, Universidad Nacional del Centro de la Provincia de Buenos Aires, Tandil, Argentina.

Davini, M. C. (2008). "Métodos de Enseñanza”. Buenos Aires, Argentina: Santillana.

Díaz Barriga, Á. (2006). "La educación en valores: Avatares del currículum formal, oculto y los temas transversales". Revista Electrónica de Investigación Educativa, 8(1), 1-15.

Gascón, Y. (2008). "El síndrome de todo menos tesis "TMT" como factor influyente en la labor investigativa". Revista Copérnico, 5(9), 46-57.

Gil Pérez, D. (1983). "Tres paradigmas básicos en la enseñanza de las ciencias". Revista de Investigación y Experiencias Didácticas, 1(1), 26-33.

Hernández Zamora, G. (2009). “Escritura académica y formación de maestros ¿Por qué no acaban la tesis?" Tiempo de educar, 10(19) 11-40.

Patrignani, M. (2019). “El acompañamiento pedagógico en el egreso universitario" (Tesis de Especialización). Universidad Nacional de La Plata, Argentina.

Pereira, C., y Di Stefano, M. (2007). “El taller de escritura en posgrado: Representaciones sociales e interacción entre pares". Revista Signos, 40(64), 405-430.

Pierella, M. P. (2014). “El ingreso a la universidad pública: diversificación de la experiencia estudiantil y procesos de afiliación a la vida institucional". Universidades, 65(60),51-62.

Ros, M., Benito, L., Germain L., y Justianovich, S. (2017). “El programa de seguimiento de trayectorias estudiantiles: Una experiencia de trabajo colectivo". En M. Ros, L. Benito, L. Germain y S. Justianovich (Eds.), Inclusión, Trayectorias Estudiantiles y Políticas Académicas en la Universidad (pp. 14-24). La Plata, Ar- 
gentina: Editorial de la Universidad Nacional de La Plata.

Rotstein De Gueller, B., y Soláns, A. P. E. (2014). “Los trabajos finales de carrera y sus autores". Estado del arte. En VI Congreso Internacional de Investigación y Práctica Profesional en Psicología XXI Jornadas de Investigación Décimo Encuentro de Investigadores en Psicología del MERCOSUR. Facultad de Psicología-Universidad de Buenos Aires, Argentina.

Santomé, J. T. (1991). "El curriculum oculto". Madrid, España: Ediciones Morata. 